\title{
The Influence of Complex Permeability on the Broadband Frequency Response of a Power Transformer
}

\author{
Steven D. Mitchell, Member, IEEE, and James S. Welsh, Member, IEEE
}

\begin{abstract}
This paper considers the effect of the complex permeability of a power transformer core on the frequency response analysis (FRA) test spectrum. Current work in FRA generally neglects the core beyond a few hundred $\mathrm{kHz}$. This research demonstrates that the effective complex permeability is significant at $1 \mathrm{MHz}$ and greater than unity at frequencies exceeding $15 \mathrm{MHz}$. The paper also shows that for broadband small signal testing, such as FRA, the low field conditions induced by the injected signal result in a relative permeability that approaches the initial permeability of the core. This ensures that the relative permeability remains approximately constant over a large range of frequencies and will have a degree of independence with respect to the amplitude of the injection voltage.
\end{abstract}

Index Terms-Condition monitoring, FRA, frequency response, high voltage, model, permeability, transformer.

\section{INTRODUCTION}

$\mathbf{F}$ REQUENCY response analysis (FRA) is a condition monitoring tool used to obtain the frequency response signature of a power transformer. This signature is topology dependent and as such can be used to detect subtle variation in the mechanical and electrical properties. The degree of variation in the frequency response can be used as a figure of merit to determine whether more invasive testing is required [1]. Whilst significant work has been published detailing methods of quantifying any observed change [2], [3], research in the area of physically interpreting the phenomena behind the change is in need of further attention [4]. The report [4] emphasizes the need for FRA research to focus particular attention on the physical modeling of a power transformer.

A transformer's core is typically constructed of grain oriented silicon steel laminations. Eddy current losses are minimized by increasing resistivity through the addition of silicon to the steel and also by reducing the lamination thickness [5]. Typical lamination thicknesses used in power transformer manufacture range from 0.23 to $0.35 \mathrm{~mm} \mathrm{[6].} \mathrm{In} \mathrm{addition,} \mathrm{the} \mathrm{use} \mathrm{of} \mathrm{grain} \mathrm{oriented}$ steels has resulted in significant reductions in a transformer's

Manuscript received November 06, 2008; revised April 07, 2009. First published December 22, 2009; current version published March 24, 2010. Paper no. TPWRD-00834-2008.

The authors are with the School of Electrical and Computer Engineering, University of Newcastle, Callaghan, NSW, Australia (e-mail: steve. mitchell@newcastle.edu.au; james.welsh@newcastle.edu.au).

Color versions of one or more of the figures in this paper are available online at http://ieeexplore.iee.org.

Digital Object Identifier 10.1109/TPWRD.2009.2036358 hysteresis losses due to the intrinsic anisotropic properties that result from aligning the steel grains [7]. The manufacture of a modern transformer's core out of thin, inherently resistive, laminations with anisotropic properties results in a magnetic circuit with high permeability at mains frequency. This permeability will attenuate with frequency as a result of magnetic skin effect [5] caused by induced eddy currents.

Research to date on physically based transformer models has typically neglected the influence of the core beyond $50 \mathrm{kHz}$ [8]-[10]. A recent paper by Abeywickrama [11] extended this frequency range and stated that the effective complex permeability of the transformer core was significant above $100 \mathrm{kHz}$ for FRA testing. A paper by Bjerkan et al. [12] measured the inductance-frequency relationship up to $600 \mathrm{kHz}$. Work by Tavner [13] on the coupling of discharge currents in the laminated steel stator core of electrical machines, showed a complex permeability greater than unity at $10 \mathrm{MHz}$ for $0.35-\mathrm{mm}$ laminations. The 0.35-mm lamination thickness used in Tavner's work is in the upper range of the lamination thickness values used in transformer manufacture. By conjecture it should be similarly applicable to transformers at even greater frequencies for the smaller lamination thicknesses. More recently, the authors have examined how significant the effective complex permeability is across the FRA spectrum [14]. The current article extends on this work.

This paper demonstrates both theoretically and experimentally that the effective complex permeability of a power transformer core is significant at $1 \mathrm{MHz}$ and greater than unity at frequencies exceeding $15 \mathrm{MHz}$. The paper also shows that there is a degree of independence with respect to the FRA test voltage due to the low field conditions. This work suggests the need to incorporate complex permeability into transformer models for the entire FRA spectrum (typically $<10 \mathrm{MHz}$ [1]). This will improve modeling accuracy and advance frequency response analysis of power transformers.

The paper is structured in the following manner. Section II provides background theory and the derivation of the effective complex permeability relationship used in the latter sections of the paper. Section III develops a physically representative model and an estimation algorithm based on the work from Section II. This model will be used in Section IV to accurately fit FRA data and determine quantitative values. Section IV also demonstrates the significance of the core's permeability at higher frequencies through comparative testing against an air cored winding. Concluding remarks are then given in Section V. 


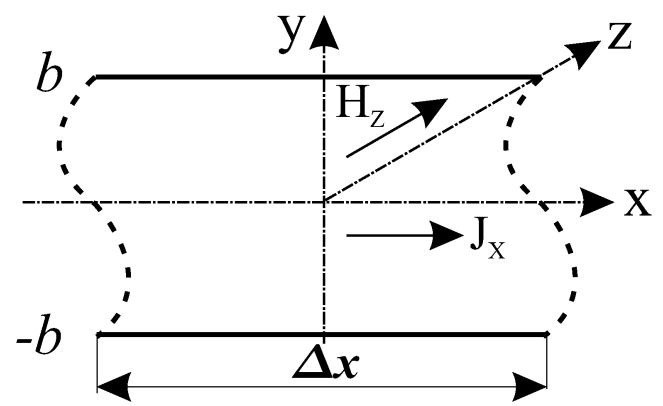

Fig. 1. One-dimensional eddy current model.

\section{THEORY}

\section{A. One-Dimensional Analysis of Eddy Currents}

The laminations in the core of a transformer have a thickness which is significantly smaller than their width and length. In addition, eddy currents tend to flow close to the conductor surface [15]. As such, the study of eddy currents in a transformer lamination can be accomplished in an approximate manner by considering current flow in one dimension only as shown in Fig. 1.

The Diffusion Equation, based on a field that is time harmonic [15], [16], quantifies how induced eddy currents will delay and attenuate the changing magnetic field inside the lamination

$$
\begin{aligned}
\frac{\partial^{2} H_{z}}{\partial y^{2}} & =j \omega \sigma \mu_{0} \mu_{r} H_{z} \\
& =\gamma^{2} H_{z}
\end{aligned}
$$

with the propagation constant

$$
\gamma=\sqrt{j \omega \sigma \mu_{0} \mu_{r}} .
$$

$\sigma$ represents conductivity, $\mu_{0}$ permeability of free space, and $\mu_{r}$ relative permeability. From (1), noting that the magnetic field intensity will not be attenuated at the surface of the lamination $(y=b)$, it can be shown [15] that

$$
\grave{H}=\frac{\gamma \Phi \cosh (\gamma b)}{2 \mu_{0} \mu_{r} \Delta_{x} \sinh (\gamma b)}
$$

where $\grave{H}$ represents the magnetic field intensity at the surface and $\Phi$ the flux in a cross sectional slice $2 b \Delta_{x}$ (refer to Fig. 1). Therefore, the space average magnetic flux density $\bar{B}$ in the $\mathrm{z}$ direction relates to the flux through the cross sectional area, i.e.,

$$
\bar{B}=\frac{\Phi}{2 b \Delta_{x}} \text {. }
$$

The effective complex permeability of a lamination is the ratio of the space average flux density to the surface magnetic field intensity. This relationship is given by

$$
\mu_{e}=\frac{\bar{B}}{\grave{H}}=\frac{\mu_{0} \mu_{r}}{\gamma b} \tanh (\gamma b) .
$$

This section outlined the derivation of the effective complex permeability. A complete derivation is provided in Appendix A.

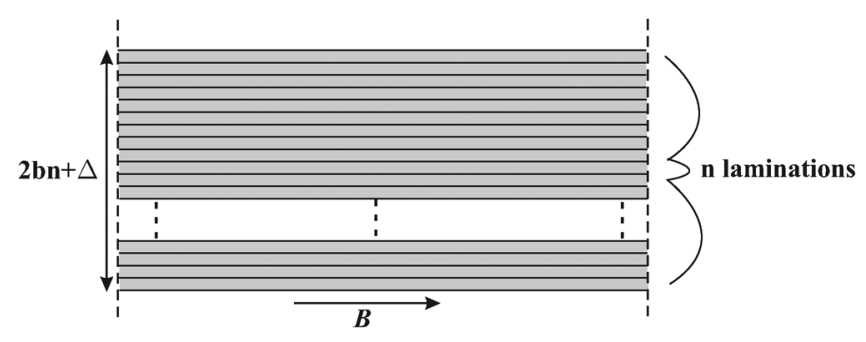

Fig. 2. Laminated transformer core; $\mathrm{n}$ laminations of $2 b$ thickness with $\Delta$ stacking excess.

\section{B. Lamination Anisotropy and Core Stacking Factor}

Typically, modern power transformers use cold rolled grain oriented silicon steel in their laminations. The grain orientation provides the laminations with anisotropic properties. It has been shown in [11] that permeability in the longitudinal (rolled) direction will be significantly greater than the permeability in the transverse direction of the lamination. As such, the paper will only consider the contribution of the longitudinal effective complex permeability.

For the modeling of a transformer core, it is not practical to consider laminations on an individual basis. This then requires a lamination stacking factor $(k)$ to be introduced such that the core can be considered as a solid block [6]. With reference to Fig. 2, a transformer core can be considered to comprise of $n$ laminations with an overall core depth of $2 b n+\Delta$ where $\Delta$ represents the stacking excess. In one dimension, the stacking factor, $k$, may then be defined as

$$
k=\frac{2 b n}{2 b n+\Delta} .
$$

From [6], the longitudinal effective complex permeability of the core is given by

$$
\mu_{e}^{*}=k\left(\mu_{e}-1\right)+1 \approx k \mu_{e} .
$$

This approximation is appropriate since lamination stacking factors for silicon steel can have values in the range of 0.95-0.98 [7]. Since permeability is typically referred to in its relative form (divided by $\mu_{0}$ ), from (5) and (7), the longitudinal effective complex relative permeability of a transformer core can be approximated by

$$
\mu_{s} \approx \frac{k \mu_{r}}{\gamma b} \tanh (\gamma b) .
$$

For the sake of brevity, the remainder of this paper will use the term effective permeability interchangeably with the term effective complex relative permeability for $\mu_{s}$. In addition, the frequency dependence of the effective permeability and its associated relationships will not be explicitly denoted in equations.

\section{Low Field Strength Relative Permeability}

FRA involves the injection of a swept sine wave or impulse signal into the windings of the transformer. The amplitude of the injected voltage is generally orders of magnitude smaller 
(a)

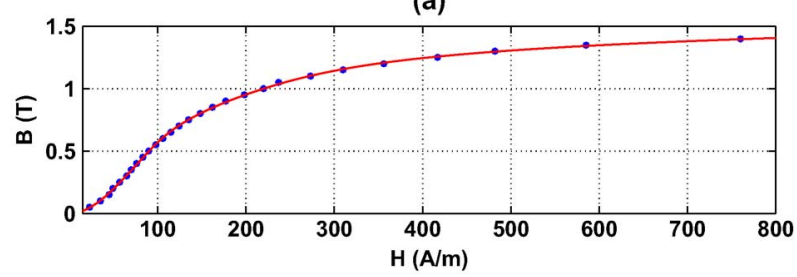

(b)

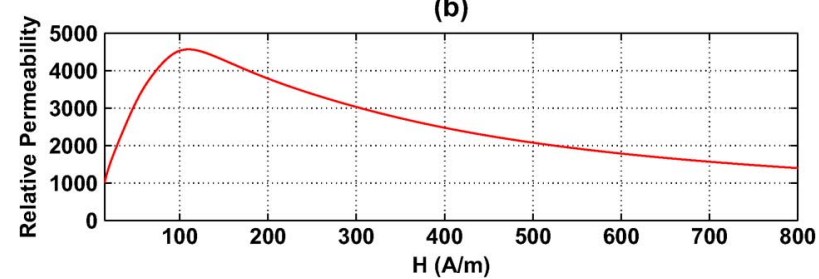

Fig. 3. (a) Typical B/H magnetization curve for $3.5 \%$ silicon steel (data from [17]). (b) Relative permeability versus magnetic field intensity based on the estimated $\mathrm{B} / \mathrm{H}$ curve.

than the nameplate operating voltage of the transformer. Examples of the maximum injection voltages used in practice are $1 V_{\mathrm{RMS}}$ for the Omicron FRAnalyzer, $2.2 V_{\mathrm{RMS}}$ for the HP89410A, 3.5 $V_{\text {RMS }}$ for the Doble M5100 and M5200 and $7 V_{\text {RMS }}$ for the Doble M5400. These voltages even at mains frequency will result in an induced magnetic field well below the operating "knee" of the B-H hysteresis curve of the core material. As such, these signals are considered to produce low field conditions. Furthermore, as the test frequency is increased a resulting increase in the inductive impedance will be observed and the influence of parasitic winding capacitance will become apparent. This will result in a further decrease in the test current and hence the associated magnetic field intensity. A change in the magnetic field intensity results in a change in the relative permeability due to the nonlinear hysteresis curve. Fig. 3 presents a typical $\mathrm{B} / \mathrm{H}$ curve for $3.5 \%$ silicon steel and the resulting relative permeability versus magnetic field intensity. However, under low field conditions, the relative permeability $\left(\mu_{r}\right)$ will approach a value equal to that of the initial permeability $\left(\mu_{i}\right)$ of the core, which is defined as being the relative permeability at zero field strength [18]

$$
\mu_{r} \approx \mu_{i}=\frac{1}{\mu_{0}} \lim _{H \rightarrow 0}\left[\frac{B}{H}\right]
$$

From (8) and (9), the core's effective permeability can be expressed for the low field condition associated with FRA

$$
\mu_{s}=\mu_{s}^{\prime}-j \mu_{s}^{\prime \prime} \approx \frac{k \mu_{i}}{\gamma b} \tanh (\gamma b)
$$

where $\mu_{s}^{\prime}$ represents the real and $\mu_{s}^{\prime \prime}$ the imaginary components of $\mu_{s}$.

\section{Estimation of the Effective Complex Relative Permeability of a Power Transformer at High Frequency}

The purpose of this paper is to show that the effective permeability of a power transformer core remains significant for the entire FRA spectrum. To demonstrate this in a theoretical

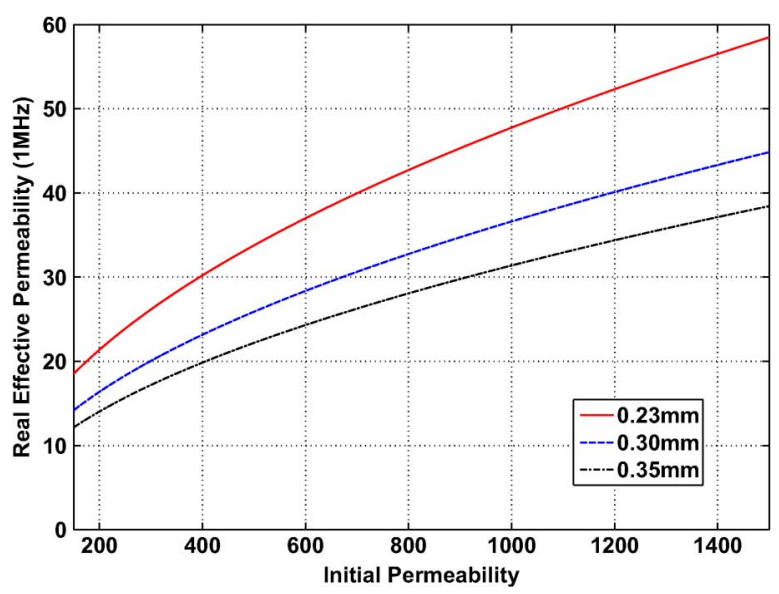

Fig. 4. Real component of the calculated effective permeability at $1 \mathrm{MHz}$ versus the initial permeability for lamination thicknesses of $0.23,0.30$, and $0.35 \mathrm{~mm}$.

TABLE I

INITIAL PERMEABILITY FOR VARIOUS GRADES of Electrical SteEl. DATA From [18]

\begin{tabular}{|c|c|}
\hline Electrical Steel Grade & $\mu_{i}$ \\
\hline \hline BSI A.253, Ferrosil 253, Losil 25 & 150 \\
\hline Magnesil & $300-1100$ \\
\hline BSI C.86, Ferrosil 86, Transil 86 & 550 \\
\hline BSI grade 62, Alphasil 62, Unisil 62 & 1000 \\
\hline Silectron, Hypersil, Transcor 3X & 1500 \\
\hline
\end{tabular}

sense, a plot of the real component of the calculated effective permeability at $1 \mathrm{MHz}$ versus initial permeability is provided in Fig. 4 via the relationship (10). The initial permeability range is based on typical values given in Table I. The lamination thickness for a power transformer generally ranges between 0.23 and $0.35 \mathrm{~mm}[6]$. A value for conductivity of $\sigma=2.1 \times 10^{6} \mathrm{~S} / \mathrm{m}$ was used which is representative of commonly used electrical steel grades-Unisil, M-2 through M-6, JIS: 30P105.

For all lamination thicknesses, the calculated minimum for the real component of the effective permeability is at least an order of magnitude above unity at $1 \mathrm{MHz}$ (Fig. 4). This implies that coupling between windings on different phases must be considered when modeling the transformer at high frequencies.

\section{E. Effective Permeability and Its Relationship With Winding Impedance}

For a winding with a permeability of unity, the inductance can be defined [19] as

$$
L_{0}=\frac{\mu_{0} N^{2} A_{c}}{l_{c}}
$$

where $l_{c}$ is the core length and $A_{c}$ the core cross sectional area. The inductance of a winding and its associated magnetic losses can be represented as an impedance which incorporates the effective permeability [20]

$$
\begin{aligned}
Z & =j w L^{\prime}+R^{\prime \prime} \\
& =j w L_{0}\left(\mu_{s}^{\prime}-j \mu_{s}^{\prime \prime}\right)
\end{aligned}
$$


where

$L^{\prime} \quad$ winding inductance;

$R^{\prime \prime}$ magnetic loss resistance;

$L_{0} \quad$ inductance at unity permeability.

From (12) and (13)

$$
\begin{aligned}
L^{\prime} & =\mu_{s}^{\prime} L_{0} \\
R^{\prime \prime} & =w \mu_{s}^{\prime \prime} L_{0} .
\end{aligned}
$$

The relationships in (14) and (15) provide a convenient method to model the complex frequency dependent relationship between the transformer windings and the laminated electrical steel core at subself resonant frequencies.

\section{EFFECtive Permeability Estimation}

To validate the premise of this paper in a practical sense, it is necessary to demonstrate that the effective permeability is significant above $1 \mathrm{MHz}$. One approach is to estimate this permeability by fitting a physically representative model, based on the theory in Section II, to the FRA data. The model parameters will only be estimated up to the self resonant frequencies such that the inductive contribution will dominate the frequency response and the influence of parasitic capacitances can be neglected.

\section{A. Subself Resonant Inductor Model}

As described in Section II-E, at frequencies below the self resonant frequency, a winding can be modeled as a series combination of its core inductance and its magnetic loss resistance. It is assumed that the copper and eddy current based losses in the windings themselves, due to skin and proximity effects, will remain small relative to the losses observed in the core, and hence can be neglected. Supporting arguments for this assumption are given in Appendix B. At higher frequencies as the permeability decays, the influence of flux leakage on the frequency response will increase. To improve the accuracy of the model, an element representing leakage inductance will complement the inductive contribution to account for quantitative variation between the winding and core cross sectional areas. The subself resonant inductor model is shown in Fig. 5, where $R^{\prime \prime}$ represents the frequency dependent magnetic loss resistance, $L^{\prime}$ the magnetic core inductance and $L_{L}$ the leakage inductance. Now

$$
V_{\mathrm{OUT}}(w)=\frac{R}{R+R^{\prime \prime}+j w L^{\prime}+j w L_{L}} V_{\mathrm{IN}}(w)
$$

where $\mathrm{R}$ represents the $50 \Omega$ FRA termination resistor. Now the transfer function, with reference to (10) and (13), becomes

$$
\hat{G}(j w)=\frac{V_{\mathrm{OUT}}(w)}{V_{\mathrm{IN}}(w)}=\frac{R}{R+j w L_{L}+j w \mu_{s} L_{0}}
$$

where

$$
\begin{aligned}
L_{0} & =\frac{\mu_{0} N^{2} A_{c}}{l_{c}} \\
L_{L} & =\frac{\mu_{0} N^{2}\left(A_{w}-A_{c}\right)}{l_{w}}
\end{aligned}
$$

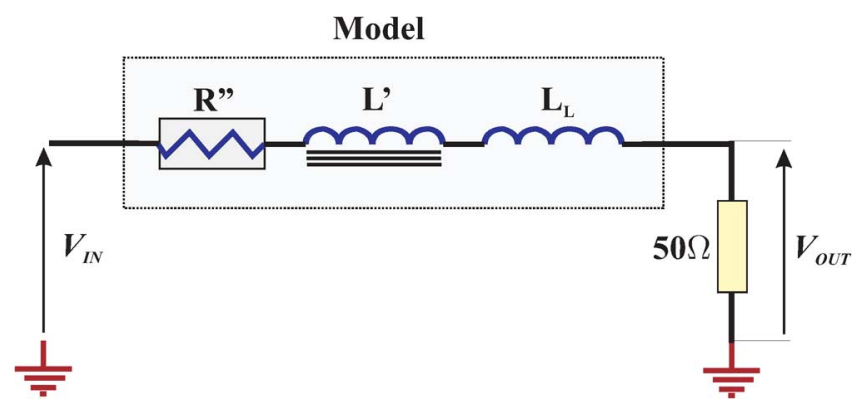

Fig. 5. Subself resonant inductor model incorporating effective permeability and leakage inductance with respect to an FRA winding test.

and

$N \quad$ number of winding turns;

$A_{c} \quad$ cross sectional area of laminated core;

$A_{w}$ cross sectional area of winding;

$l_{c} \quad$ length of laminated core;

$l_{w} \quad$ length of winding.

\section{B. Estimation Algorithm}

To estimate the effective permeability with respect to frequency, a gradient based search algorithm is implemented. This algorithm determines the best fit between the proposed model and the FRA data by finding the parameters that result in the lowest cost. The minimum cost, $J$, is calculated using the cumulative residual between corresponding model and data frequency points

$$
J=\min \left\|\log _{10}\left(\frac{\hat{G}(j w)}{G(j w)}\right)\right\|^{2}
$$

where $G(j w)$ is the observed frequency response and $\hat{G}(j w)$ is the model frequency response. Using the knowledge of $R, L_{0}$, and $L_{L}$, an estimate for the initial permeability, $\mu_{i}$, and conductivity, $\sigma$, can be determined by

$$
\left[\hat{\mu}_{i}, \hat{\sigma}\right]=\arg \min _{\boldsymbol{\mu}_{\mathbf{i}}, \boldsymbol{\sigma}}\left\|\log _{10}\left(\frac{\hat{G}(j w)}{G(j w)}\right)\right\|^{2}
$$

where $\hat{\mu}_{i}$ represents the estimated initial permeability and $\hat{\sigma}$, the estimated conductivity. Utilising the estimated parameters together with the given parameters, an estimate of the effective permeability as a function of frequency can be obtained. This algorithm is implemented in Section IV-D.

\section{RESUlts}

This section experimentally verifies the work developed in the previous sections. In particular, this section will demonstrate that:

1) under FRA testing conditions, relative permeability approaches the initial permeability of the lamination material; 
2) the effective permeability is significant above $1 \mathrm{MHz}$;

3) the effective permeability is greater than unity at frequencies exceeding $15 \mathrm{MHz}$.

\section{A. Broadband Frequency Response Analysis Test Bed}

A test bed is used to investigate the effective permeability of a power transformer's core with respect to frequency. It is important that the test bed is representative of a power transformer under similar test conditions. Recall that the permeability of a transformer's core is governed by the lamination material properties and the level of excitation. To ensure its practical relevance to power transformer FRA, 0.35-mm laminations (the upper bound for a typical transformer lamination [6]) is utilised as the core material for all experiments. With respect to the level of excitation, the levels induced by a typical FRA transformer test will be many orders of magnitude less than those observed under normal operating conditions. As such, it is proposed that the level of excitation induced in the test bed will be of a similar magnitude to that observed by a typical power transformer during an FRA test.

One of the primary goals of the experimental test bed is to restrict the resonant behaviour to frequencies above those of interest. This is accomplished through the use of a single-layer winding. A single-layer winding possesses no interlayer and little interturn capacitance. As such, it does not possess the low frequency self resonance feature of multilayer windings. If the distributed winding to ground and measurement cable shunt capacitances are kept to a minimum, it is possible to obtain a frequency response that is completely dominated by the inductive response for frequencies greater than $1 \mathrm{MHz}$. Fig. 6 depicts the two configurations used for all of the experiments in the paper.

Fig. 7 shows the veracity of this experimental approach when tested with the 200-turn, 0.35 -mm laminated core inductor. It is readily apparent that resonance for this winding configuration occurs at frequencies greater than $1 \mathrm{MHz}$. Hence, this configuration is only utilized for frequencies less than $1 \mathrm{MHz}$. In a similar fashion, it can also be shown that the frequency response for the test bed's 5-turn winding remains resonance free at frequencies less than $15 \mathrm{MHz}$. As an aside, the observed resonant behavior in Fig. 7 is due to the resonant interaction between the distributed inductance and the shunt capacitances to ground of both the winding and measurement cables.

An inductive response over a large range of frequencies provides an ideal platform to observe the effective permeability. This approach facilitates direct comparisons between a core constructed with laminations, typical of a power transformer, and that of an air cored equivalent, across a large range of frequencies.

\section{B. Practical Confirmation of the Low Field Assumption}

The relative permeability of a transformer core is governed by its B-H curve. Since the magnetic field intensity is current dependent, an injection voltage of constant amplitude results in a change in relative permeability with frequency as the inductive impedance of a winding increases. In Section II-C, the conjecture was made that, due to the small injection voltages used by FRA test equipment relative to nameplate voltage, the generated magnetic field intensity could be considered as a low field

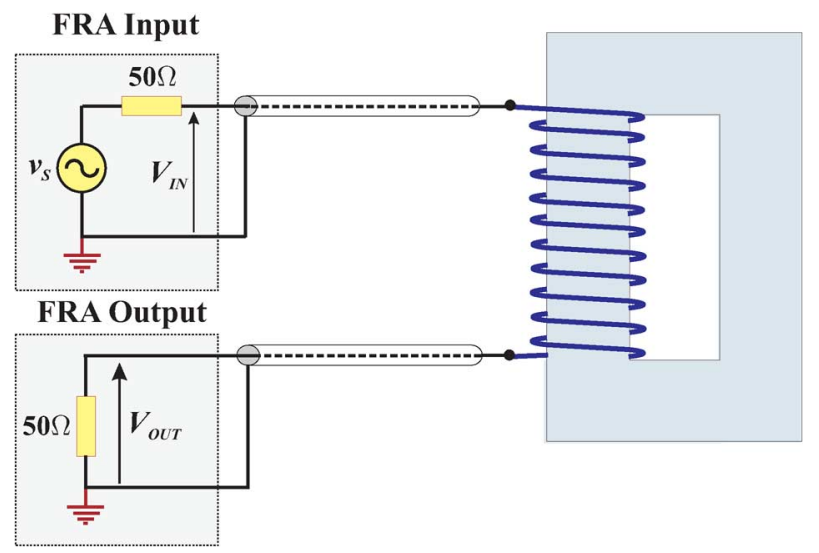

Fig. 6. FRA test bed.

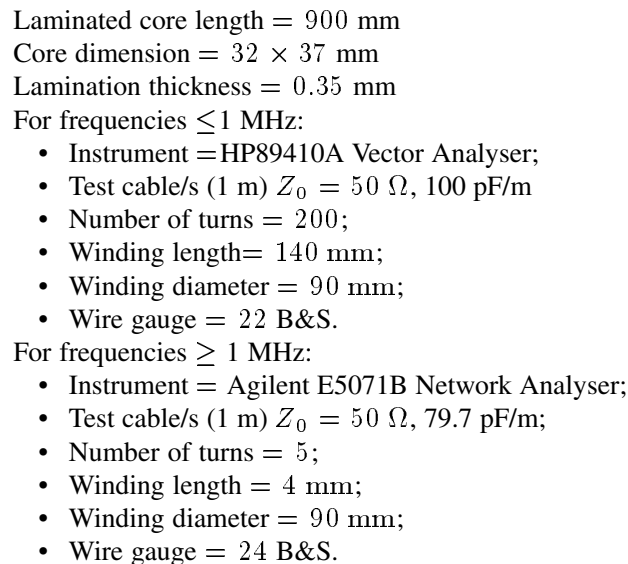

- Wire gauge $=24 \mathrm{~B} \& \mathrm{~S}$

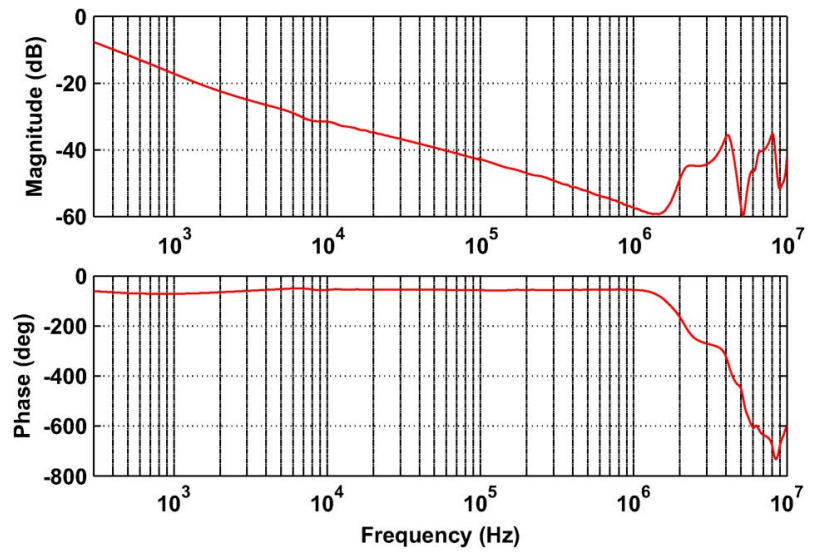

Fig. 7. Frequency response of the experimental test bed utilizing the singlelayer 200-turn laminated core inductor for frequencies up to $10 \mathrm{MHz}$.

condition, that is $H \rightarrow 0$, over the FRA spectrum. As such, the assumption was made that the relative permeability will approximate that of the initial permeability and remain relatively constant across the test frequency spectrum. The validation of this assumption is important from a modeling perspective since it simplifies the complex nonlinear relationship between an applied test voltage and the resulting relative permeability.

To confirm the low field conjecture in a practical setting, it is necessary to show that the frequency dependent winding inductance demonstrates a level of independence with respect to the FRA injection voltage. This will be indicative of a constant relative permeability as per (9) and (10). 

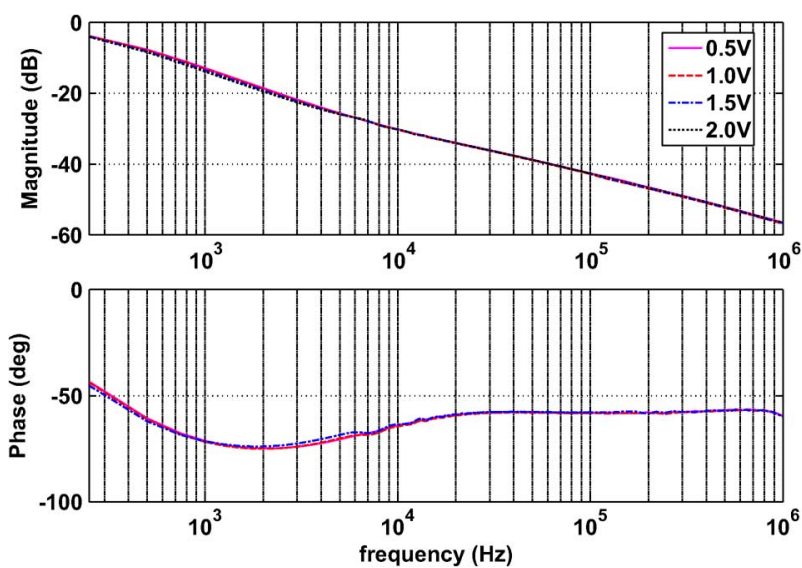

Fig. 8. Frequency response of the single-layer 200-turn laminated core inductor using several test voltages $\left(0.5 V_{\mathrm{RMS}}, 1.0 V_{\mathrm{RMS}}, 1.5 V_{\mathrm{RMS}}, 2.0 V_{\mathrm{RMS}}\right)$.

Experiments were conducted with a HP89410A vector analyzer using a range of test voltages from 0.5 to $2.0 \mathrm{Vrms}$. These voltages are representative of those used by FRA testing authorities in the field. For example, the OMICRON FRAnalyzer utilizes a 1 Vrms injection voltage and Doble's M5100 and M5200 utilize a 3.5 Vrms injection voltage. Furthermore, it is important to ensure that the test bed core is in a demagnetised condition prior to conducting the test. It has been shown [21] that the magnetic viscosity of electrical steel will reach its steady state condition after a period of approximately $24 \mathrm{~h}$. To ensure the validity of the test results, experiments were conducted on the core after it had been left in a de-energized state for a period greater than 24 hours. Experiments were then conducted in a sequence from lowest to highest injection voltage to ensure that the resulting core magnetization level related only to the current test, and was not due to residual effects.

The experimental results are presented in Fig. 8. Only subtle variations in the frequency response can be observed, hence demonstrating independence with respect to FRA injection voltages. This is highlighted in Fig. 9, where the range of frequency has been restricted to show these small variations in magnitude for each injection voltage in finer detail. This supports the assumption that, under FRA testing conditions, relative permeability will approximate the initial permeability.

Small errors that may be introduced due to this approximation can be minimised by conducting FRA tests using lower amplitude injection voltages. However, there will be a trade off between the low field response and the measurement device sensitivity i.e. acceptable SNR of the frequency response.

\section{Practical Demonstration of High Frequency Effective Permeability}

To confirm that the effective permeability is still significant at $1 \mathrm{MHz}$, an FRA test was conducted on a 200-turn single-layer inductor with a core constructed from $0.35-\mathrm{mm}$ silicon steel laminations. To provide a permeability reference of unity, an identical test was conducted on the winding in an air cored configuration. To complete the experiment, a test on the winding with a solid steel core was conducted to demonstrate the effec-

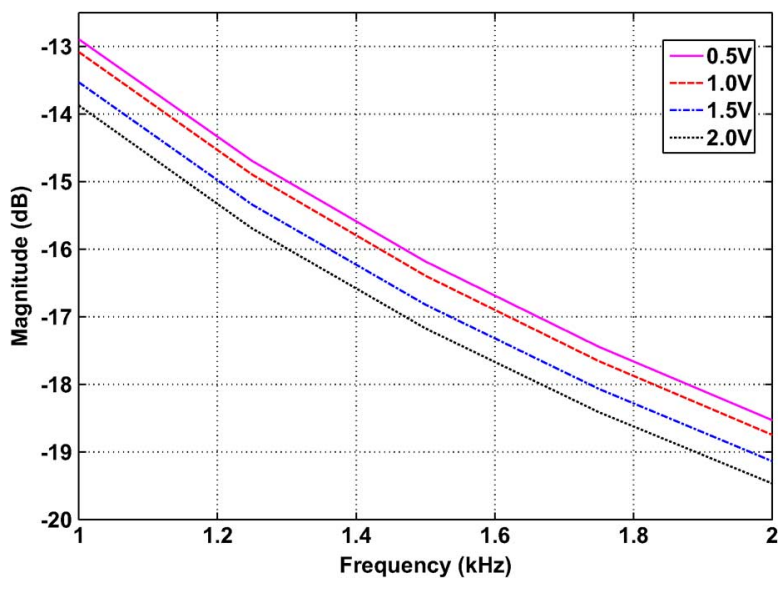

Fig. 9. Zoomed in view of the frequency response of the single-layer 200turn laminated core inductor using several test voltages $\left(0.5 V_{\mathrm{RMS}}, 1.0 V_{\mathrm{RMS}}\right.$, $1.5 V_{\mathrm{RMS}}, 2.0 V_{\mathrm{RMS}}$ ).
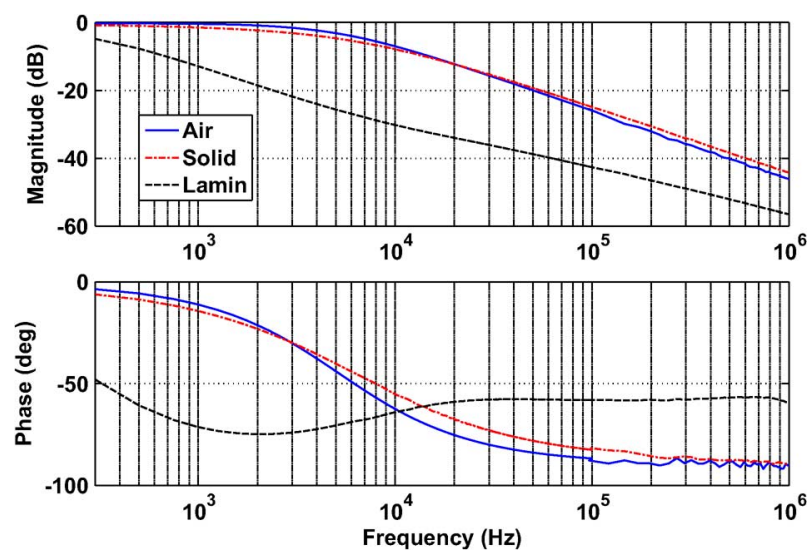

Fig. 10. Frequency responses of the 200-turn single-layer inductor with an air core, solid steel core and a laminated electrical steel core $(0.35-\mathrm{mm}$ laminations).

tive permeability tending to unity. The results are presented in Fig. 10.

In Fig. 10, it can be observed across all frequencies that the level of attenuation for the laminated core is significantly greater than that observed by the reference air core, despite having an identical winding. This clearly indicates that the effects of the magnetic core are the dominating factor in the frequency response. The frequency responses shown in Fig. 10 support the theoretical results in Section II-D. The laminated core configuration has significantly more attenuation than the air cored version at $1 \mathrm{MHz}$. This is indicative of a larger inductance which, since the same winding is used for all tests, corresponds to a permeability greater than unity.

The winding with the solid steel core demonstrates the impact of magnetic skin effect. Within a few kilohertz, skin depth $(\delta=$ $\left.\sqrt{2 / w \sigma \mu_{0} \mu_{r}}\right)$ is significantly smaller than the core dimensions with the effective permeability tending to unity and essentially following the air cored winding frequency response as expected.

\section{Estimation Algorithm for Effective Permeability}

In Section III, an estimation algorithm was proposed in order to fit a subself resonant inductor model to FRA data. Using 
the test configuration shown in Fig. 6, with test frequencies not exceeding $1 \mathrm{MHz}$, FRA data was recorded. To accommodate material dependent variation in the conductivity for silicon steel (M235-35 $\sigma=1.69 \times 10^{6} \mathrm{~S} / \mathrm{m}$, Ferrosil $86 \sigma=$ $1.82 \times 10^{6} \mathrm{~S} / \mathrm{m}$, JIS: $\left.30 \mathrm{P} 105 \sigma=2.1 \times 10^{6} \mathrm{~S} / \mathrm{m}\right)$, a constraint was placed on the conductivity parameter in the parameter estimation algorithm. The lower and upper constraints were set to $\sigma_{L}=1.5 \times 10^{6} \mathrm{~S} / \mathrm{m}$ and $\sigma_{H}=2.1 \times 10^{6} \mathrm{~S} / \mathrm{m}$. The stacking factor was approximated to be $k=0.95$. In conjunction with the test bed data specified in Fig. 6, the initial permeability and conductivity is estimated by the minimisation of the accumulated residual error between the frequency response of the model and data sets.

Note that for the winding under test

$$
\begin{aligned}
A_{c} & =\text { Laminated core cross sectional area } \\
& =1.184 \times 10^{-3} \mathrm{~m}^{2} \\
A_{w} & =\text { Winding cross sectional area }=6.36 \times 10^{-3} \mathrm{~m}^{2} .
\end{aligned}
$$

Now from (11)

$$
L_{0}=\frac{\mu_{0} N^{2} A_{c}}{l_{c}}=66 \times 10^{-6} H .
$$

By considering the residual cross-sectional area of the winding to approximate the leakage inductance component

$$
L_{L} \approx \frac{\mu_{0} N^{2}\left(A_{w}-A_{c}\right)}{l_{w}}=1.9 \times 10^{-3} H .
$$

From (2) and (9), the propagation constant can be found in terms of the initial permeability

$$
\gamma \approx \sqrt{j \omega \sigma \mu_{0} \mu_{i}} .
$$

Substituting (24) into (10), noting that lamination thickness is equal to $2 b$

$$
\mu_{s} \approx \frac{k \mu_{i}}{\gamma b} \tanh (\gamma b)
$$

Finally substituting (22), (23), and (25) into (17), the winding model transfer function is

$$
\hat{G}(j w)=\frac{50}{50+j w\left[0.0019+0.36 \frac{\mu_{i}}{\gamma} \tanh \left(1.8 \times 10^{-4} \times \gamma\right)\right]} .
$$

The transfer function (26) is utilized in the gradient search based algorithm as described in Section III-B using the cost function (21). The estimated values for $\mu_{i}$ and $\sigma$ are 835 and $1.72 \times$ $10^{6} \mathrm{~S} / \mathrm{m}$ respectively. The resulting frequency response of the model (26) and the recorded FRA data is presented in Fig. 11. As observed in this plot, the estimated model response is quite accurate in both magnitude and phase for four decades of frequency. This degree of accuracy supports the modeling approach and provides the necessary confidence in the estimated effective permeability (refer to Fig. 12). From Fig. 12, the value of the effective permeability at $1 \mathrm{MHz}$ is $\mu_{s}=33-33 j$. Based on this
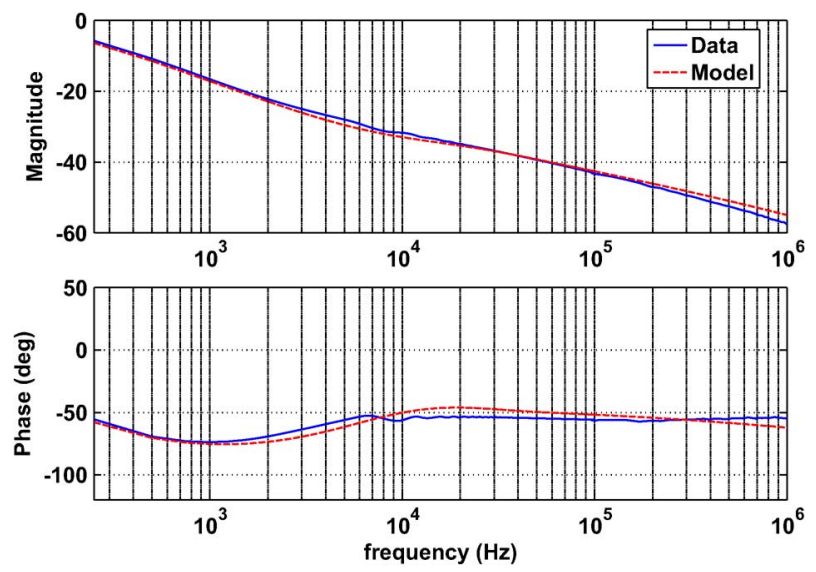

Fig. 11. Subself resonant inductor model fitting to FRA data of the 200-turn single-layer inductor. The test voltage was $1.5 V_{\mathrm{RMS}}$.

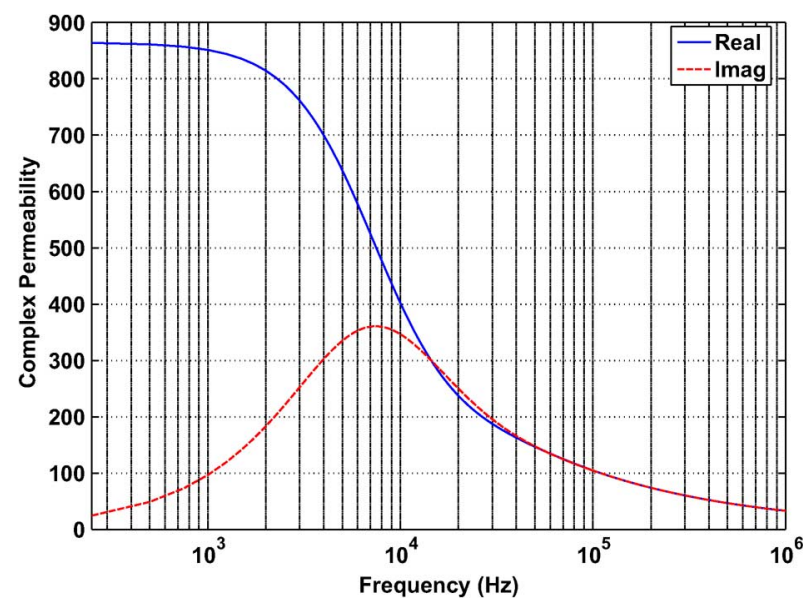

Fig. 12. Estimated effective permeability based on the subself resonant inductor model fitting; $u^{\prime}$ (real component) and $u^{\prime \prime}$ (imaginary component).

estimate, it can be concluded that there exists an effective permeability over thirty times greater than unity at $1 \mathrm{MHz}$ for a lamination thickness of $0.35 \mathrm{~mm}$. As previously discussed, this lamination thickness is typically the largest used in power transformer manufacture [6], hence even larger permeabilities would be obtained with smaller lamination thicknesses.

It should be noted that the effective permeability observed at high frequency is not large in comparison with the permeabilities observed under normal operating conditions at mains frequency. However, these values are significant when considering the subtleties involved in frequency response analysis and the associated coupling between windings of a three phase transformer.

\section{E. An Effective Permeability of Unity}

At frequencies greater than several megahertz, the measurement equipment itself becomes influential in the observed FRA test. The measurement cables, potentially several metres in length, can have a distributed capacitance of $100 \mathrm{pF} / \mathrm{m}$. In addition, the dimensions of the transformer windings under test are such that transmission line effects will not be negligible. As testing frequencies increase above a couple of megahertz 


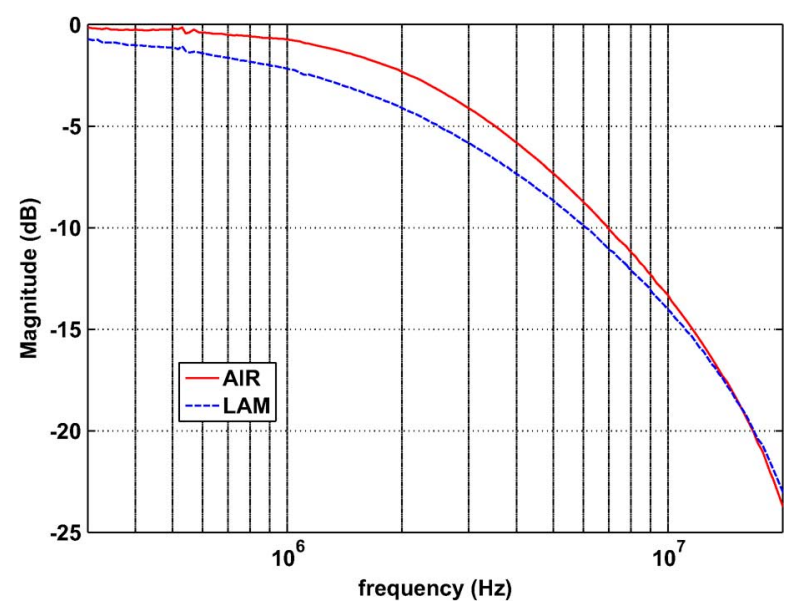

Fig. 13. High-frequency FRA tests on the 5-turn single-layer inductor with an air core and a laminated $0.35-\mathrm{mm}$ electrical steel core.

the usefulness of FRA testing becomes questionable and it becomes more difficult to accurately record and assess. The recommended diagnostic range for a M5400 FRA test device is $20 \mathrm{~Hz}-2 \mathrm{MHz}$ [22]. However, in order for this investigation to be rigorous, experiments were conducted to locate the frequency at which the effective permeability of a $0.35-\mathrm{mm}$ lamination silicon steel core is approximately one.

To achieve this without self resonant interference and to minimize transmission line effects, a winding with five single-layer turns was used. The test was conducted using an Agilent E5071B Network Analyzer in the same configuration shown in Fig. 6. The use of the network analyzer facilitates a testing platform capable of taking into account the measurement equipment and associated high frequency transmission line effects. It does this by characterizing the winding under test in terms of incident and reflected power waves, and determining the associated scattering parameters.

An experiment was conducted to determine the forward voltage gain scattering parameter [23] which represents the high frequency transfer function. The high-frequency response for the test winding is shown in Fig. 13.

The results in Fig. 13 quantitatively demonstrate that the laminated core winding has a larger inductive impedance than the corresponding air cored winding, at frequencies exceeding 15 $\mathrm{MHz}$. As all the other parameters are equal, the effective permeability must also be greater than unity at frequencies larger than $15 \mathrm{MHz}$. It should also be noted that the frequency where the effective permeability reaches unity would be even higher for smaller lamination thickness values $(<0.35 \mathrm{~mm})$ used in power transformer manufacture.

\section{CONCLUSION}

The paper proposed that an FRA test on a power transformer could be considered as a low field condition where the relative permeability approaches the initial permeability. As such, the relative permeability remains approximately constant across the frequency spectrum for a fixed amplitude injection voltage despite the highly nonlinear nature of the hysteresis curve.
This results in a degree of independence with respect to the applied FRA test voltage. The paper validated this approximation by performing FRA tests on a winding using a range of relevant injection voltages. The results showed only very small differences between the frequency response at each voltage. The paper also showed that the effective complex relative permeability for a power transformer remains significant for frequencies exceeding $1 \mathrm{MHz}$. This was demonstrated by comparing the level of inductive attenuation at $1 \mathrm{MHz}$ for a winding with and without the laminated core. A model was also fitted to the frequency response of the winding to confirm the theoretical conjectures. Finally, for experimental rigor, the frequency response of a winding using the $0.35-\mathrm{mm}$ laminations, was compared to an air cored version to demonstrate that an effective complex relative permeability of unity occurred at a frequency greater than $15 \mathrm{MHz}$.

\section{APPENDIX A \\ ANALYSIS OF EDDY CURRENTS IN ONE DIMENSION}

Since a transformer's lamination thickness is significantly smaller than its width and length, and eddy currents tend to flow close to the surface, analysis of eddy currents in a lamination can be approximated to have current flow in one dimension only (refer Fig. 1). From Faraday's law for electromagnetic induction

$$
\nabla \mathbf{x} \mathbf{E}=-\frac{\partial \mathbf{B}}{\partial t}
$$

Relating magnetic field intensity $\mathbf{H}$ to magnetic flux density $\mathbf{B}$ through the permeability relationship

$$
\nabla \mathbf{x E}=-\mu_{0} \mu_{r} \frac{\partial \mathbf{H}}{\partial t} .
$$

Furthermore, electric field strength $\mathbf{E}$ can be replaced with current density $\mathbf{J}$ through the conductivity relationship

$$
\nabla \mathbf{x} \mathbf{J}=-\sigma \mu_{0} \mu_{r} \frac{\partial \mathbf{H}}{\partial t}
$$

where $\sigma$ represents conductivity, $\mu_{0}$ permeability of free space and $\mu_{r}$ relative permeability. With reference to Fig. 1, the 1-D analysis is performed such that the magnetic field is applied in the $\mathrm{z}$ direction with the induced eddy currents flowing in the $\mathrm{x}$ direction. This reduces (29) to

$$
\frac{\partial J_{x}}{\partial y}=\sigma \mu_{0} \mu_{r} \frac{\partial H_{z}}{\partial t} .
$$

From Ampére's law, neglecting displacement current

$$
\nabla \mathbf{x H}=\mathbf{J}
$$

Once again utilizing the 1-D model, (31) reduces to

$$
\frac{\partial H_{z}}{\partial y}=J_{x}
$$

Differentiating (32)

$$
\frac{\partial^{2} H_{z}}{\partial y^{2}}=\frac{\partial J_{x}}{\partial y}
$$


Substituting (30) into (33) leads to the derivation of the Diffusion Equation [15]

$$
\frac{\partial^{2} H_{z}}{\partial y^{2}}=\sigma \mu_{0} \mu_{r} \frac{\partial H_{z}}{\partial t}
$$

This is an important relationship which equates the delay and attenuation in the changing magnetic field, inside the lamination, to the induced eddy currents. Assuming that the field is time harmonic [16], the Diffusion Equation may be written as

$$
\begin{aligned}
\frac{\partial^{2} H_{z}}{\partial y^{2}} & =j \omega \sigma \mu_{0} \mu_{r} H_{z} \\
& =\gamma^{2} H_{z}
\end{aligned}
$$

where $\gamma$ is the propagation constant given by

$$
\gamma=\sqrt{j \omega \sigma \mu_{0} \mu_{r}} .
$$

Since the solution is in one dimension, the subscript $z$ denoting direction will be removed from $H$ in all further references. Solving for $H$

$$
H=\beta \cosh (\gamma y) \quad \text { where } \beta=\text { constant. }
$$

With reference to Fig. 1, deriving the flux in a cross sectional slice $2 b \Delta_{x}$, we have

$$
\begin{aligned}
\Phi & =\int_{-b}^{b} \mu_{0} \mu_{r} H \Delta_{x} d y \\
& =\int_{-b}^{b} \mu_{0} \mu_{r} \Delta_{x} \beta \cosh (\gamma y) d y \\
& =\frac{2 \mu_{0} \mu_{r} \Delta_{x} \beta \sinh (\gamma b)}{\gamma} .
\end{aligned}
$$

Solving (38) for $\beta$

$$
\beta=\frac{\gamma \Phi}{2 \mu_{0} \mu_{r} \Delta_{x} \sinh (\gamma b)}
$$

Substituting (39) into (37)

$$
H=\frac{\gamma \Phi \cosh (\gamma y)}{2 \mu_{0} \mu_{r} \Delta_{x} \sinh (\gamma b)} .
$$

As the magnetic field intensity will not be attenuated at the surface of the lamination $(y=b)$

$$
\grave{H}=\frac{\gamma \Phi \cosh (\gamma b)}{2 \mu_{0} \mu_{r} \Delta_{x} \sinh (\gamma b)}
$$

where $\grave{H}$ represents the magnetic field intensity at the surface. The space average magnetic flux density $\bar{B}$ in the $\mathrm{z}$ direction relates the flux through the cross sectional area as

$$
\bar{B}=\frac{\Phi}{2 b \Delta_{x}} .
$$

The effective complex permeability of a lamination is the ratio of the space average flux density to the surface magnetic field intensity. This relationship is

$$
\mu_{e}=\frac{\bar{B}}{\grave{H}}=\frac{\mu_{0} \mu_{r}}{\gamma b} \tanh (\gamma b) .
$$

\section{APPENDIX B}

\section{EDDY CURRENT LOSSES IN THE WINDING}

In Section III-A, the assumption was made that eddy current based losses in the winding are small relative to core losses and can therefore be neglected. This appendix justifies this assertion.

\section{A. Dowell Method}

Eddy current effects within the windings themselves will also introduce losses. These losses can be classified into two categories: skin and proximity effect.

Skin effect is due to the magnetic field generated by the current in the conductor. This has the effect of increasing the current density near the conductor surface relative to its centre, which results in an increase in the effective resistance.

Proximity effect is where the current in adjacent conductors generate magnetic fields which induce circulating eddy currents in neighboring conductors. This will impact on the conductors current distribution, and therefore will also increase the effective resistance.

An analytical estimate for winding losses can be made using the Dowell Method [24]. If the conductors are closely packed, each layer of a winding will approximate the geometry of a conductor foil, once again reducing the problem to a 1-D model.

For the ac resistance due to skin effect [25]

$$
R_{s e}=\frac{R_{d c} \xi}{2}\left[\frac{\sinh \xi+\sin \xi}{\cosh \xi-\cos \xi}\right]
$$

where

$$
\xi=\frac{d \sqrt{\pi}}{2 \delta}
$$

$d$ is the conductor diameter and

$$
\delta=\frac{1}{\sqrt{\pi f \mu \sigma}}
$$

is the skin depth.

Considering proximity effect, the ac resistance [25] for the $m$ th layer is

$$
R_{p e}=\frac{R_{d c} \xi}{2}\left[(2 m-1)^{2} \frac{\sinh \xi-\sin \xi}{\cosh \xi+\cos \xi}\right] .
$$

If it is assumed that the magnetic field from the other conductors is uniform across the conductor cross section, an orthogonal relationship exists between the skin and proximity effect [26] The two effects can be decoupled and an estimate for the total eddy current losses can be determined through the addition of both effects (44) and (45), hence

$$
R_{a c}=R_{s e}+R_{p e} .
$$




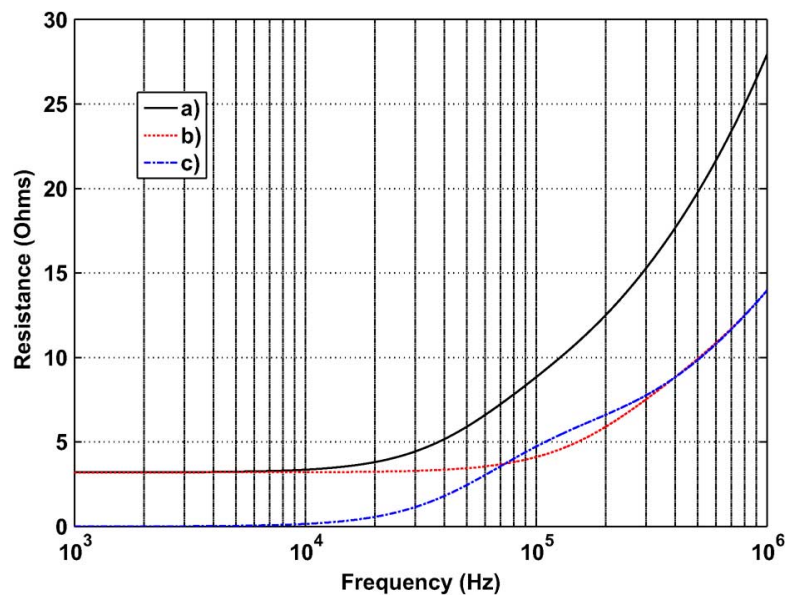

Fig. 14. Estimated ac resistance for the 200-turn single-layer test bed winding. (a) Total ac resistance; (b) skin effect resistance contribution; and (c) proximity effect resistance contribution.

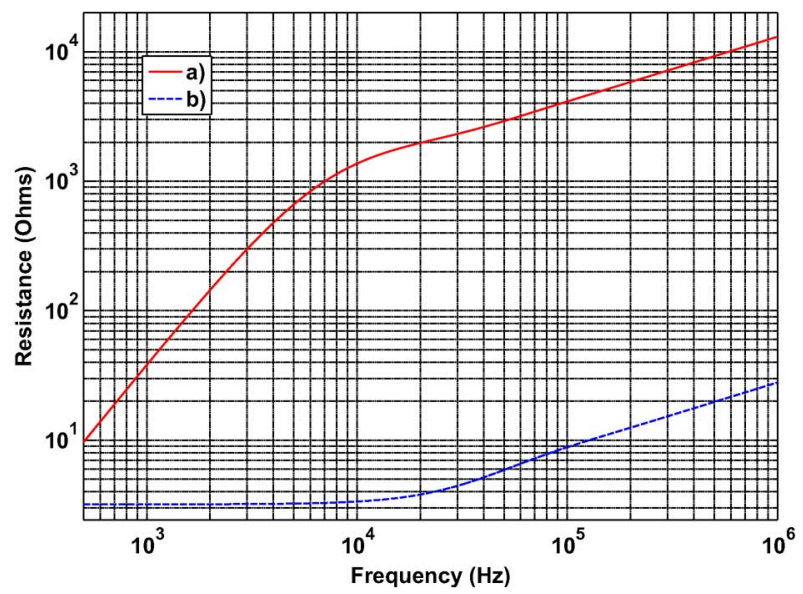

Fig. 15. Comparison between the estimated model of the magnetic loss resistance to the calculated winding loss due to proximity and skin effect. (a) Estimated model magnetic loss resistance and (b) estimated winding ac resistance.

\section{B. Test Bed Winding Eddy Current Loss Estimate}

With reference to (44), (45) and (46), the winding loss can be estimated for the combined influence of proximity and skin effect. These losses are calculated for the winding utilized in the test bed for frequencies up to $1 \mathrm{MHz}$ (refer to Fig. 6 for the winding details). The estimated results for the winding loss, Fig. 14, indicate only a modest increase in resistance with frequency. With reference to (45), it can be observed that proximity effect losses are dependent on the winding layer being considered. Since the test bed winding was constructed as a single layer to limit low frequency resonant modes, an additional effect was the minimization of proximity effect losses.

In order to compare the estimated winding losses and the losses due to the core, it is necessary to determine an estimate for the core losses. This is accomplished by extracting the real component of the inductor impedance from the model estimate in Fig. 11. From (15) where $R^{\prime \prime}$ is the magnetic loss resistance. The magnetic loss resistance can then be compared to the winding loss resistance, Fig. 15. Above a few kHz, the magnetic loss resistance is orders of magnitude larger than the estimated winding contribution. Allowing for approximation errors, the significant difference in magnitude of the loss components indicates that it is appropriate to consider the winding resistance negligible for this particular experimental configuration.

\section{REFERENCES}

[1] C. L. Sweetser, "Experience with sweep frequency response analysis (SFRA) measurements," in Proc. IEEE/PES Transformers Committee, Vancouver, BC, Canada, 2002.

[2] S. Ryder, "Methods for comparing frequency response analysis measurements," in Proc. Conf. Record 2002 IEEE Int. Symp. Electrical Insulation, Apr. 2002, pp. 187-190.

[3] J. Welsh, C. Rojas, and S. Mitchell, "Wideband parametric identification of a power transformer," in Proc. Universities Power Engineering Conf. (AUPEC), Australasian, Dec. 2007, pp. 1-6.

[4] "Mechanical condition assessment of transformer windings using frequency response analysis (FRA)," ELECTRA-CIGRE WG A2.26 Rep. vol. 228, Oct. 2006.

[5] G. R. Slemon and A. Straughen, Electric Machines. Reading, MA: Addision-Wesley, 1982.

[6] S. V. Kulkarni and S. A. Khaparde, Transformer Engineering: Design and Practice. New York: Marcel Dekker, 2004.

[7] M. J. Heathcote, The $J \&$ \& Pransformer Book, 12th ed. New York: Reed Educational and Professional Publishing Ltd, 1998.

[8] Z. Wang, P. Crossley, and K. Cornick, "A simulation model for propagation of partial discharge pulses in transformers," in Proc. Int. Conf. Power System Technology (POWERCON), Aug. 1998, vol. 1, pp. 151-155.

[9] G. Gharehpetian, H. Mohseni, and K. Moller, "Hybrid modelling of inhomogeneous transformer winding for very fast transient overvoltage studies," IEEE Trans. Power Del., vol. 13, no. 1, Jan. 1998.

[10] E. Rahimpour, J. Christian, K. Feser, and H. Mohseni, "Transfer function method to diagnose axial displacement and radial deformation of transformer windings," IEEE Trans. Power Del., vol. 18, no. 2, pp. 493-505, Apr. 2003.

[11] K. Abeywickrama, T. Daszczynski, Y. Serdyuk, and S. Gubanski, "Determination of complex permeability of silicon steel for use in high-frequency modeling of power transformers," IEEE Trans. Magn., vol. 44, no. 4, pp. 438-444, Apr. 2008.

[12] E. Bjerkan, H. Hoidalen, and O. Moreau, "Importance of a proper iron core representation in high frequency power transformer models," in Proc. Int. Symp. High Voltage Engineering, Beijing, China, 2005.

[13] P. Tavner and R. Jackson, "Coupling of discharge currents between conductors of electrical machines owing to laminated steel core," IEE Proc. B, Elect. Power Applic., vol. 135, no. 6, Nov. 1988.

[14] S. D. Mitchell and J. S. Welsh, "Permeability and its influence on the broadband frequency response of a power transformer," in Proc. Asia-Pacific Power and Energy Engineering Conf. (APPEEC), Wuhan, China, 2009.

[15] R. L. Stoll, The Analysis of Eddy Currents. New York: Clarendon, 1974.

[16] D. K. Cheng, Field and Wave Electromagnetics. Reading, MA: Addision-Wesley, 1983.

[17] I. Boldea and S. A. Nasar, The Induction Machine Handbook. Boca Raton, FL: CRC Press, 2001.

[18] K. L. Kaiser, Electromagnetic Compatibility Handbook. Boca Raton, FL: CRC Press, 2004.

[19] M. A. Plonus, Applied Electromagnetics, P. D. Nalle and M. Gardner, Eds. New York: McGraw-Hill, 1988.

[20] E. C. Snelling, Soft Ferrites. London, U.K.: Iliffe Books Ltd, 1969.

[21] N. Abeywickrama, Y. Serdyuk, and S. Gubanski, "Effect of core magnetization on frequency response analysis (fra) of power transformers," IEEE Trans. Power Del., vol. 23, no. 3, pp. 1432-1438, Jul. 2008.

[22] M5400 Sweep Frequency Response Analyser-Technical Specification Doble Eng. Co., Tech. Rep.

[23] R. Ludwig and P. Bretchko, RF Circuit Design, M. Horton, Ed. Upper Saddle River, NJ: Prentice-Hall, 2000.

[24] P. Dowell, "Effects of eddy currents in transformer windings," Proc. IEE, vol. 113, pp. 1387-1394, 1966.

$$
R^{\prime \prime}=w \mu_{s}^{\prime \prime} L_{0}
$$


[25] J. Ferreira, "Improved analytical modeling of conductive losses in magnetic components," IEEE Trans. Power Electron., vol. 9, no. 1, pp. 127-131, Jan. 1994.

[26] X. Nan and C. Sullivan, An Improved Calculation of Proximity-Effect Loss in High-Frequency Windings of Round Conductors vol. 2, pp. 853-860, Jun. 2003.

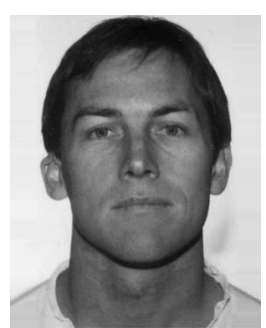

Steven D. Mitchell (M'07) was born on December 24, 1969, in Newcastle, Australia. He received the B.E. degree (Hons.) in electrical engineering from the University of Newcastle, Callaghan, NSW, Australia, in 1996. He is currently pursuing the Ph.D. degree with focus on power transformer modeling for partial discharge localization and frequency response analysis.

He is a Lecturer in the School of Electrical Engineering and Computer Science, University of Newcastle. His previous industry experience was gained through work as a Design Engineer in heavy manufacturing and underground mine control/protection equipment design between 1988 and 2000, and as a Research Engineer at the University of Newcastle between 2000 and 2008.

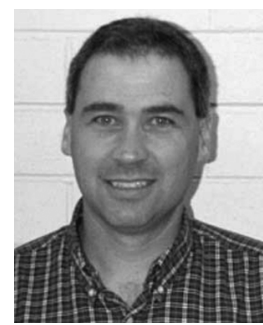

James S. Welsh (M'00) was born in Maitland, Australia, in 1965. He received the B.E. degree (Hons. I) in electrical engineering in 1997 and the Ph.D. degree, with focus on ill-conditioning problems arising in system identification, in 2004, both from The University of Newcastle, Callaghan, NSW, Australia.

During the last several years, he has been actively involved in research projects at the Centre for Complex Dynamic Systems and Control, The University of Newcastle, including Powertrain Control, Model Predictive Control, and System Identification Toolboxes. His research interests include auto-tuning, system identification, and process control. He is currently a Senior Lecturer in the School of Electrical Engineering and Computer Science, The University of Newcastle. 